\title{
Design of Milkotester for Fat and CLR Measurement using Arduino Microcontroller
}

\author{
Abhishek M. Aware ${ }^{1}$, Ujwala A. Kshirsagar (Belorkar) ${ }^{2}$ \\ P.G. Student, Electronics and Telecommunication Department, HVPM's College of Engineering and Technology, \\ Amravati, India ${ }^{1}$ \\ Professor, Electronics and Telecommunication Department, HVPM's College of Engineering and Technology, \\ Amravati, India ${ }^{2}$
}

\begin{abstract}
This paper introduces the design and working of arduino microcontroller for measuring the FAT and CLR. In many dairy farms, computer aided control of physiological and sanitary parameters are already used and lead to a productivity increase and the elimination of some tedious operations. Embedded Technology is now in its prime and the wealth of knowledge available is mind-blowing. An embedded system can be defined as a control system or computer system designed to perform a specific task. Embedded systems are playing important roles in our lives every day, even though they might not necessarily be visible. Here I describes one of the applications of embedded system MILKOTESTER. It is Small compact, embedded in a single unit, requires less power and measure milk parameters like SNF (Solid but Not FAT), FAT, CLR with less cost. Also with the help of IOT (Internet of Things) process the milk industry should be able to send the real time reading information of milk to the government so that it helps to overcome the illegal things such as milk quality during the production of milk pocket.
\end{abstract}

Keywords: Fast analysis-allows a large number of measurement to be done,Simple and light weight design, Low cost, Low power consumption, Easy to operate.

\section{INTRODUCTION}

With the initiatives of National Dairy Development Board (NDDB), out of 70,000 dairy cooperative societies in the country, around 26000 are using Electronic Milko-Testers (EMT) and around 2500 are using the PC connected electronic milko-tester machines (known as Automatic Milk Collection Systems - AMCS). These systems introduced very satisfactory milk collection methods and facilitated immediate payments to farmers based on the quality and quantity of milk delivered.

The success of these systems coupled with inexpensive connectivity opportunity offered by Internet, motivated the CEG-IIMA to enhance the PC at the Automatic Milk Collection Systems (AMCS) into a Dairy Information Services Kiosk (DISK) and offer an extensive knowledge and service delivery mechanism through a Dairy Portal. The DISK when used with a Dairy Portal of the Union, enhances the scope of services that would benefit the farmers as well as the dairy industries.

The system I propose as a project proposal is an electronic milk tester (Milk analysis embedded system / Milkotester) which can be used to find the quality of the milk samples by measuring more than one parameters at once. The targeted beneficiaries of this project proposal would be both the milk farmers and the industries in whole. The farmers would be benefiting in the sense that they would get a just pricing and they cannot get cheated by the system, since manipulating it would be harder than manipulating a physical measuring scale. The industry would benefit because this can be used as a cheap alternative to the cost prohibitive and non-user friendly meters available abroad. This project proposal would allow for anindigenous developed tool that can be used as a complete solution for this.

\section{LITERATURE REVIEW}

A proof of concept application using Information and Communication Technology (ICT) in the dairy sector was developed by the Centre for Electronics Governance at the Indian Institute of Management, Ahmedabad (CEG-IIMA). The application aims at helping the dairy farmers with timely messages and educating them on the care for their milch cattle and enhances the production of quality milk. It also aims at assisting the dairy unions in effectively scheduling and organizing the veterinary, artificial insemination, cattle feed and other related services. The application uses Personal Computers at the milk collection Centres of the Dairy Cooperative Societies (DCS) having connectivity to an Internet Service Provider (ISP). The application includes two components - a Dairy Portal (DP) and a Dairy Information Services Kiosk (DISK). This paper presents IIMA-CEG's efforts to design and implement the DISK and Dairy Portal. [2] 
This "Milk Producer Group Resource Book" is part of a series of practical field guides for people working in smallscale dairying in developing countries. These field guides are produced by the Animal Production and Health Division of the Food and Agriculture Organization (FAO) of the United Nations. Milk producers can increase their income and utilise their skills and resources better if they are working in groups. This book aims to promote the organization of small-scale milk collection and processing as a sustainable, income-generating activity for household food security. It also tries to be a means to improving the safety, quantity and quality of milk and milk products available for consumers in developing countries. The intended readers are (future) leaders of milk producer groups, extension workers, project staff and group promoters who are working to set up milk producer groups, and those developing already existing groups at village level in rural areas. Some excellent FAO booklets exist on working with small groups (see information sources and references). They complement this book which has been written specifically for milk producer groups. This Milk producer group resource book aims to play a role in poverty alleviation in developing countries in a gender sensitive and sustainable way. [5]

\section{PROBLEM DEFINITION}

The milk analysis embedded system is an important product for milk collection parameters such as weight, FAT \& CLR. In this project proposal I will propose a new method for measuring liquid density and also a method for measuring the amount of fat in it.

A device will setup based on this method for measuring the milk quality. The device will observed to have almost negligible amount of delay for measuring and processing. The fat measurement will found to be very accurate and precise within the range of sample values.

Hence to design an embedded system with IOT is the main task of my project proposal. Various design scheme and circuit will be investigated for embedded system circuit.

\section{PROPOSED WORK}

After having seen the old and implemented technologies and the current "newer technology" in dairy sciences, I came up with a completely new method for measuring the milk parameters. And here I put forward my proposed methodology for such a milk testing instrument.

So, as far as I have seen, to calculate the quality of a milk sample we need at the least two variables in the form of the specific gravity of the sample and its fat content. But the greatest problem that we would face would be the actual measurement of the CLR of the sample, due to the above mentioned fact of unavailability of affordable technology which can be used as a method. Rather than use a small improvement over a lactometer, like an "Auto-CLR", which does nothing in improving the accuracy of the system, I propose a completely new method of measuring liquid density. To measure a physical quantity we must make use of one of the physical properties. All the previous methods use one of the properties. Ultrasonic method used the reflectance of the sound, and oscillating U-tube used the measure of natural harmonic oscillations of a body. I plan to use a fairly unused property in measuring the density that is the gauge pressure created by a column of liquid. Mathematically, we know that the pressure exerted by a column of fluid at a particular depth is dependent only on the depth of the point, the density of liquid and the acceleration due to gravity (which is a constant). Or,

$$
\mathrm{P}=\mathrm{H} \times \rho \times \mathrm{g}
$$

Since we already know that acceleration due to gravity is a constant, and if we can measure the pressure at a fixed depth of the liquid, we can find the density of the liquid from the above equation by one simple calculation.

Once the density of the liquid is known the CLR can be directly calculated by dividing the density by the density of water. This will give a direct specific gravity reading and will require no temperature corrections as done in lactometers and Auto-CLRs. The second variable to measure is the fat content. Since the existing method of the light based scattering analysis of samples is both fast and accurate. And unlike Gerber method it will not use up the sample and it can be kept for further analysis without any wastage. Here I plan to use a paired IR LED and a phototransistor as a module. The system would be set up with the receiver and transmitter separated by a small distance inside the given sample. Fat molecules tend to specifically absorb certain bands of IR radiation.

\section{DESIGN METHODOLOGY}

\section{Arduino microcontroller:}

Arduino is a flexible programmable hardware platform designed for artists, designers, tinkerers, and themakers of things. Arduino's little, blue circuit board, mythically taking its name from a local pub in Italy, has in a very short time motivated a new generation of DIYers of all ages to make all manner of wildprojects found anywhere from the hallowed grounds of our universities to the scorching desert sands ofa particularly infamous yearly arts festival and just about everywhere in between. Usually these Arduino based projects require little to no programming skills or 
knowledge of electronics theory, and more oftenthan not, this handiness is simply picked up along the way.Central to the Arduino interface board, shown in Figure 1, is an onboard microcontroller - thinkof it as a little computer on a chip.

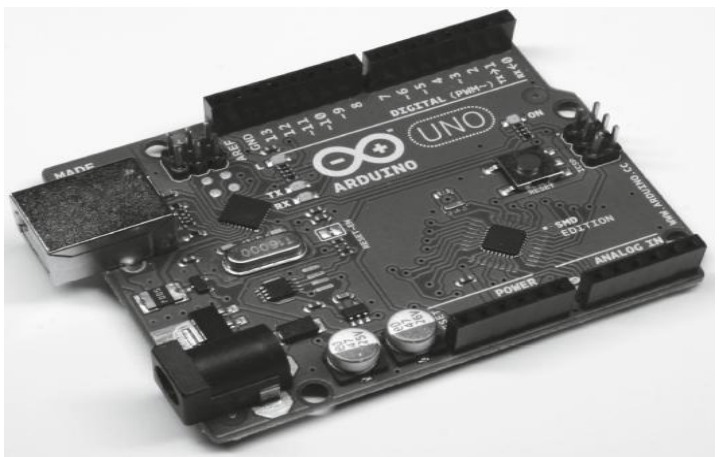

Figure1: The Arduino Uno interface boar

This microcontroller comes from a company called Atmel and the chip is known as an AVR. It is slow in modern terms, running at only $16 \mathrm{Mhz}$ with an 8 -bit core, and has a very limited amount ofavailable memory, with $32 \mathrm{kilobytes}$ of storage and 2 kilobytes of random access memory. The interfaceboard is known for its rather quirky design-just ask the die-hards about standardized pin spacing-butit also epitomizes the minimalist mantra of only making things as complicated as they absolutely need tobe. Its design is not entirely new or revolutionary, beginning with a curious merger of two, off-the-shelfreference designs, one for an inexpensive microcontroller and the other for a USB-to-serial converter,with a handful of other useful components all wrapped up in a single board. Its predecessors include thevenerable BASIC Stamp, which got its start as early as 1992, as well as the OOPic, Basic ATOM, BASICX24,and the PICAXE.

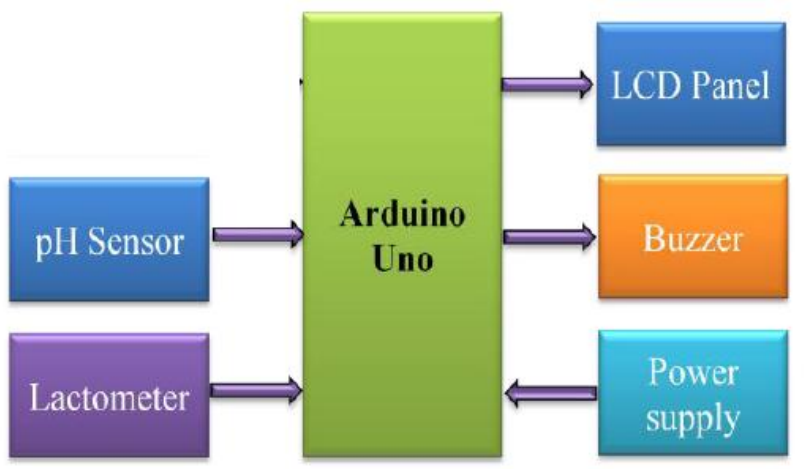

Figure2: Block diagram of Milk parameter measurement system

\section{FAT measurement by MILKOTESTER:}

The scattering of a beam of light by the fat globules present in the homogenized milk is the principle in the Milkotester. The amount of light scattered by the milk sample is a measure of the fat content in the milk.

\section{Construction:}

A high intensity LED is used as a light source. The light beam is made to pass through the sample solution contained in the test tube. A LDR is placed exactly on the opposite side of the test tube to detect the amount of light passing through the test tube unscattered. To obtain maximum sensitivity the test tube is covered in wooden shield which has opening only for LED and LDR(Light dependent resistor) to pass through.

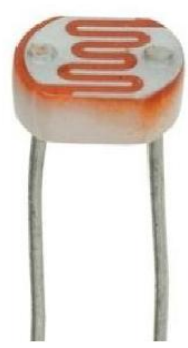

Figure3: Light dependent resister 


\section{Working:}

This solution is then made to pass through a syringe needle to disperse the fat globules homogeneously throughout the sample the solution. Then this sample solution is introduced in the test tube and beam of light is passed through it. The more the fat content in the milk, more will be the amount of light scattered by the sample. Thus the light reaching the LDR will vary with the fat in the milk, thus the change in the resistance of LDR is indication of the fat content .The circuit is calibrated using standards with sample of known fat values .The voltages are also adjusted within a range suitable for the MUX following this circuitry.

The range of fat content acceptable to the market is fixed by the government to ensure the customer of the nutrition value of the milk.

- $\quad$ For cow's milk fat should be in range of 3.5 to 4.5 percent.

- $\quad$ For buffalo's it is 6-7 percent.

Thus it is possible to incorporate a program in the microcontroller to give the alarm for the milk which doesn't fall in these ranges and so milk is rejected .This would also need a selector switch to enable the microcontroller to know whether it is cow or buffalo milk

\section{CLR measurement:}

The specific gravity of the milk is measured using a 'Lactometer' and the temperature deviation of milk is taken into consideration and correction applied, the lactometer is called Correct Lactometer Reading (CLR). The Auto CLR is an instrument incorporating electronics to observe the lactometer reading .It is a patented instrument of its manufacturer 'Solid State Technologies'. In this case the manual process is preserved, only electronics is 'added' to it make observation error free and apply the temperature correction automatically.

\section{Construction:}

A $120 \mathrm{ml}$ cylinder is used to contain the milk sample. The lactometer is suspended in the freely movable and vertically arranged cylinder. The original position oflactometer is obtained by taking water in the cylinder and allowing the lactometer to attain a undisturbed position in which it shows ' 0 ' on the scale.

\section{Working:}

Take $120 \mathrm{ml}$ of milk is in cylinder. The lactometer moves in a vertical direction and attains a fixed floating position. The lactometer reading is calibrated on scale on lactometer itself .The reading on the lactometer corresponding to the level of the milk gives the lactometer reading. But in Auto CLR we measure this vertical movement electronically. The upper tip of the lactometer is attached to the float of the type is used in motorcycles to indicate fuel level. This float moves vertically along with motion of lactometer. Using a strain gauge attached to the float, the resistance change is calibrated as a measure of the lactometer reading.

\section{CONCLUSIONS}

This paper is excellent blending of bio-chemistry and electronics engineering. The milk collection parameters such as FAT \& CLR are measured by this system gives same results as the existing systems which are more costly than the developed one.

\section{REFERENCES}

[1] RupakChakravarty, a paper on" IT at Milk collection centers in Cooperative Diaries: The National Dairy Development Board Experience", pp.37-47.

[2] SubhashBhatnagar, "Empowering Dairy Farmers: A Portal and Dairy Information and Services Kiosk"

[3] Wolf, W.H., "Hardware-software co-design of embedded systems", IEEE Jul 1994, Page(s): 967 - 989

[4] Harold Macy, W.B. Combs \& C.H. Eckles," Milk \& Milk Products", TMH, Fourth edition 1990.

[5] JurjenDraaijer, "Milk Producer group Resource Book a practical guide to assist milk producer groups", Pp.37-40. 